\title{
Focus on the training characteristics of Excellent Engineers, Building a new teaching mode of Digital Electronic Technology Based on CKDI
}

\author{
Jingjing Yang ${ }^{1, a}$, Xiaogang $\mathrm{Mu}^{2, \mathrm{~b},{ }^{*}}$ and Yanfei $\mathrm{Liu}^{3, \mathrm{c}}$ \\ ${ }^{1}$ Xi'an Research Institute of High Technology, Xi'an 710025, China \\ 2Xi'an Research Institute of High Technology, Xi'an 710025, China \\ ${ }^{3}$ Xi'an Research Institute of High Technology, Xi'an 710025, China \\ ayangjingjing8405@sina.com, bmuxg2001@163.com, cbbmcu@126.com
}

Keywords: CKDI, Excellent Engineers, Digital electronic technology

\begin{abstract}
Based on the practice teaching reform as the breach, CKDI as theoretical guidance, IBOARD electronic experimental box and the FPGA development board as the carrier, a new teaching content system of digital electronic technology course is built carefully. It optimizes the integration of teaching content, highlights the frontier of teaching content, the practice of teaching process and the exploratory of teaching activities. The research interests of the excellent engineers have been stimulated, and the practical ability and innovation ability of the excellent engineers have been enhanced.
\end{abstract}

\section{Preface}

The outstanding engineers training plan is launched by education ministry and Chinese engineering academy in 2010. The target which is based on the plan as the breakthrough point promotes the engineering education reform and innovation, comprehensively improves the quality of Chinese engineering education personnel training, and promotes our country from big engineering education to strong engineering education ${ }^{[1]}$. At the same time, the implementation of the plan marks the beginning of education reform which is centered on the training of students' innovation and practice ability in the field of higher education ${ }^{[2]}$.

Based on the practice teaching reform as the breach, CKDI (C represents Conceive idea, $\mathrm{K}$ represents Knowledge, D represents Design, I represents Implement) as the theoretical guidance, strengthen and cultivating innovative thinking, innovative spirit, team consciousness and practical ability of outstanding engineers as the key point, IBOARD electronic experimental box and the FPGA development board as the carrier, the new teaching pattern of "digital electronic technology" course is built carefully.

\section{Break the boundaries of theory, experiment and practice, optimize the content system from the perspective of the system, and highlight "new, refined and live".}

"Digital electronic technology" course spans in the basis of "circuit analysis", "analog electronic technology" and other important professional basic courses and "microcomputer principle and interface technology", "the principle and application of single-chip", "DSP technology" and other important professional courses. It plays an important role between the two most courses. With the development of integration and complexity of the semiconductor integrated circuit, digital electronic technology has been developed from small to very large scale integrated circuit, from the fixed function device to the development of large-scale programmable devices, and has penetrated into every aspect of human production and life. The curriculum of digital electronic technology should also keep up with the times, update the content of the curriculum and highlight "new, refined and live".

New-Curriculum content should keep up with the times, closely follow the trend of digital electronic technology development, combine the actual requirement of excellent engineers. The 
curriculum content system is optimized from a more systematic perspective [3].

Fine-Select a typical system that covers important knowledge points. It helps excellent engineers building up the connection of knowledge points from an application perspective through the introduction of typical system. It improves the excellent engineers' ability to solve practical problems in the digital circuit.

Live-Starting from the knowledge source, the cause and effect of knowledge points, the status in the knowledge point spectrum diagram, the connection with other knowledge points and the application of knowledge points are explained clearly. It improves the living and learning ability of excellent engineers.

\section{Following the cognitive rules of the excellent engineers, build the example teaching method, and highlight" typical, comprehensive and diversity".}

According to the theoretical and practical curriculum characteristics, this paper constructs an example teaching method based on the training needs of excellent engineers. This method is a kind of teaching method on the basis of the engineering example. Its main characteristic is based on an engineering example for traction, with excellent engineers as the main body, with the teacher as the leading factor, to solve the problem as the main line, around the discussion of the engineering example, analysis to expand the teaching content gradually.

According to the principle of "strong engineering background, strong knowledge comprehensive application and the diversity of implement method ", engineering cases, typical cases of life, research projects and competition content which are related to this course are selected carefully as classroom teaching cases. It not only stimulates the interest of the excellent engineers, but also broadens the horizon. In analyzing the process of the case, teachers organize and guide excellent engineers to interpret and discuss the case of specific problems, and make excellent engineers fully discussion and published their own opinions. Teachers guide, judge and summary timely ${ }^{[4]}$.

By analyzing cases of related knowledge, the purpose that using knowledge to solve practical problems is achieved. It mobilizes the enthusiasm and initiative of excellent engineers, realizes the integration of theory and practice. At the same time, the multi-knowledge point fusion, cross-curricular knowledge fusion and knowledge integration have been realized with the case as the carrier.

\section{Putting forward the new teaching concept of CKDI, and construct a new teaching mode of "trinity", and highlight "autonomy, inquiry and engineering".}

For excellent engineers of narrow knowledge, poor initiative ability, the teaching concept of CKDI (C represents Conceive idea, K represents Knowledge, D represents Design, I represents Implement) which with the knowledge, ability and quality that product development needs as the goal, with the engineering practice as the background is proposed. It integrates idea, knowledge, design and the integration of engineering application ability into the teaching process. The systematic curriculum system of mutual contact and support is adopted to train the excellent engineers, so as to enrich their experiences in design, development and active learning in the learning and practice environment. It not only encourages excellent engineers to innovate personalized, but also makes the creative potential ability of excellent engineers to fully display based on the engineering practice. It make ups the insufficient of lack of the knowledge correlation in traditional teaching, achieves the purpose of building innovation atmosphere and stimulating innovation interest, promotes the integration growth of knowledge, ability and quality.

According to the specific requirements of this course that CKDI teaching concept and training plan of excellent engineers proposes, the new teaching mode of trinity which consists of theory teaching, simulation proof and physical structures is constructed. It lays a solid foundation for engineering practice ability and innovative spirit training of excellent engineers. It makes the system method throughout, takes the IBOARD electronic experimental box and FPGA software two lines to propel. It explains both traditional bottom-up design patterns and modernization of 
top-down design method. Excellent engineers study the digital circuits and FPGA simultaneously. Firstly, excellent engineers use the FPGA software to complete the simulation validation of functional circuit design. Secondly, excellent engineers use the IBOARD circuit experiment box to build circuit. This makes the excellent engineers master the EDA realization method of modern digital system on the basis of mastering the general digital system hardware implementation. This not only deepens the understanding depth of basic knowledge and circuit by using FPGA technique, but also practices the practical ability [5]. It cultivates the practice ability and the team cooperation spirit of the excellent engineers, forms the exploring habits, improves the self-organization and management ability, lays a solid foundation for engineering practice ability and innovative spirit of excellent engineers.

\section{Summary}

The independent learning ability, engineering practice ability and innovative spirit of the excellent engineers have been improved significantly, and excellent engineers have achieved remarkable achievements in various competitions. Excellent engineers can clearly understand the teaching purpose, highly endorse the engineering, comprehensive, probing characteristics of course case, generally believe the engineering background of case, the independent choice of task promote the learning interest and initiative. The curriculum cultivates the ability of "innovative consciousness, integrated design, research exploration and engineering practice" of excellent engineers. In recent years, more than $50 \%$ of the excellent engineers participate in the subject competitions such as national undergraduate electronic design competition, the national college students' smart car competition and so on after learning courses. The obtained results show that the ability of autonomic learning, engineering practice ability and innovation spirit of excellent engineers has been improved significantly after implementation of new teaching mode and new method that this paper proposed.

\section{References}

[1] Huangyishen, Dongchenchen and so on. Explore the construction of CDIO practice platform for excellent engineers [J]. Journal of Zhejiang University of technology (social science edition), 2013(4).

[2] Luole, Sunwei and so on. To promote the development of education training for excellent engineer by MCU model car competition $[\mathrm{J}]$. Experimental technology and management,2015(2).

[3] Loushuqin and so on. Digital electronic technology foundation system method[M].Beijing: China Machine Press,2014.

[4] Liufengchun and so on. Examples of teaching cases of "electronic technology" course engineering teaching [J]. Journal of electrical and electronic teaching,2012(4).

[5] Zhangli and so on. An exploratory teaching of electrical and electronic technology courses [J]. Technology education innovation,2009(9). 